\title{
Design of Z-pinch and Dense Plasma Focus Powered Vehicles
}

\author{
Tara Polsgrove, Sharon Fincher, Robert B. Adams \\ National Aeronautics and Space Administration, George C. Marshall Space Flight Center, MSFC, AL \\ Jason Cassibry, Ross Cortez, Matthew Turner \\ University of Alabama in Huntsville, Huntsville, AL \\ C. Daphne Maples \\ Qualis Corporation, Huntsville, AL \\ Janie N. Miernik, Geoffrey N. Statham \\ ERC Inc., Huntsville, AL \\ Leo Fabisinski \\ International Space Systems, Inc., Huntsville, AL \\ John Santarius \\ University of Wisconsin \\ Tom Percy \\ SAIC, Inc., Huntsville, AL
}

Z-pinch and Dense Plasma Focus (DPF) are two promising techniques for bringing fusion power to the field of in-space propulsion. A design team comprising of engineers and scientists from UAHuntsville, NASA's George C. Marshall Space Flight Center and the University of Wisconsin developed concept vehicles for a crewed round trip mission to Mars and an interstellar precursor mission. Outlined in this paper are vehicle concepts, complete with conceptual analysis of the mission profile, operations, structural and thermal analysis and power/avionics design. Additionally engineering design of the thruster itself is included. The design efforts adds greatly to the fidelity of estimates for power density (alpha) and overall performance for these thruster concepts.

\section{Introduction}

Fusion-based propulsion can enable fast interplanetary transportation. Magneto-inertial fusion (MIF) is an approach which has been shown to potentially lead to a low cost, small reactor for fusion break even ${ }^{1}$. The Z-Pinch/dense plasma focus method is an MIF concept in which a column of gas is converted to plasma and then compressed to thermonuclear conditions by an axial current (I 100 MA). Recent advancements in experiments and the theoretical understanding of this concept suggest favorable scaling of fusion power output yield as $\mathrm{I}^{42}$. This document presents a conceptual design of a Z-Pinch fusion propulsion system and a vehicle for human exploration. A more comprehensive general review of the project ${ }^{3}$ and a comparison of the proposed Z-pinch system to other fusion systems $^{4}$ are also available.

The purpose of this study is to apply Z-Pinch fusion principals to the design of a propulsion system for an interplanetary spacecraft. This study took four steps in service of that objective; these steps are identified below.

1. Z-Pinch Modeling and Analysis: There is a wealth of literature characterizing Z-Pinch physics and existing Z-Pinch physics models, e.g. ${ }^{1,5,6}$. In order to be useful in engineering analysis, simplified Z-Pinch fusion thermodynamic models are required to give propulsion engineers the quantity of plasma, plasma temperature, rate of expansion, etc. The study team developed these models in this study.

2. Propulsion Modeling and Analysis: While the Z-Pinch models characterize the fusion process itself, propulsion models calculate the parameters that characterize the propulsion system (thrust, specific impulse, etc.) The study team developed a Z-Pinch propulsion model and used it to determine the best 
values for pulse rate, amount of propellant per pulse, and mixture ratio of the D-T and liner materials as well as the resulting thrust and specific impulse of the system.

3. Mission Analysis: Several potential missions were studied. Trajectory analysis using data from the propulsion model was used to determine the duration of the propulsion burns, the amount of propellant expended to complete each mission considered.

4. Vehicle Design: To understand the applicability of Z-Pinch propulsion to interplanetary travel, it is necessary to design a concept vehicle that uses it - the propulsion system significantly impacts the design of the electrical, thermal control, avionics and structural subsystems of a vehicle. The study team developed a conceptual design of an interplanetary vehicle that transports crew and cargo to Mars and back and can be reused for other missions. Several aspects of this vehicle are based on a previous crewed fusion vehicle study - the Human Outer Planet Exploration (HOPE) Magnetized Target Fusion (MTF) vehicle. Portions of the vehicle design were used outright and others were modified from the MTF design in order to maintain comparability.

\section{Z-Pinch Operation}

The approach investigated in this study involves the use of a confinement scheme known as a Z-Pinch, which falls under the MIF regime. The premise of a Z-Pinch is to run very large currents (Megampere scale) through a plasma over short timescales $(200 \mathrm{~ns})$. The magnetic field resulting from the large current then compresses the plasma to fusion conditions. This plasma formation is widely used in the field of Nuclear Weapons Effects (NWE) testing in the defense industry, as well as fusion energy research. Facilities of note include the Z Machine at Sandia National Laboratories (SNL) and MAGPIE at Imperial College, London. For a fusion propulsion system, the Z-Pinch is formed using annular nozzles with Deuterium-Tritium (D-T) fuel in the innermost nozzle and a Lithium mixture containing Lithium-6/7 in the outermost nozzle. The configuration would be focused in a conical manner so the D-T fuel and Lithium-6/7 mixture meet at a specific point that acts as a cathode so that the lithium mixture can serve as a current return path to complete the circuit, as shown in Figures 1 and 2.

In addition to serving as a current return path, the lithium liner also serves as a radiation shield. The advantage to this configuration is the reaction between neutrons and Lithium- 6 resulting in the production of Tritium, thus adding further fuel to the fusion reaction, and boosting the energy output. In utilizing this method of fusion for propulsion, high thrusts and/or specific impulse can be produced.

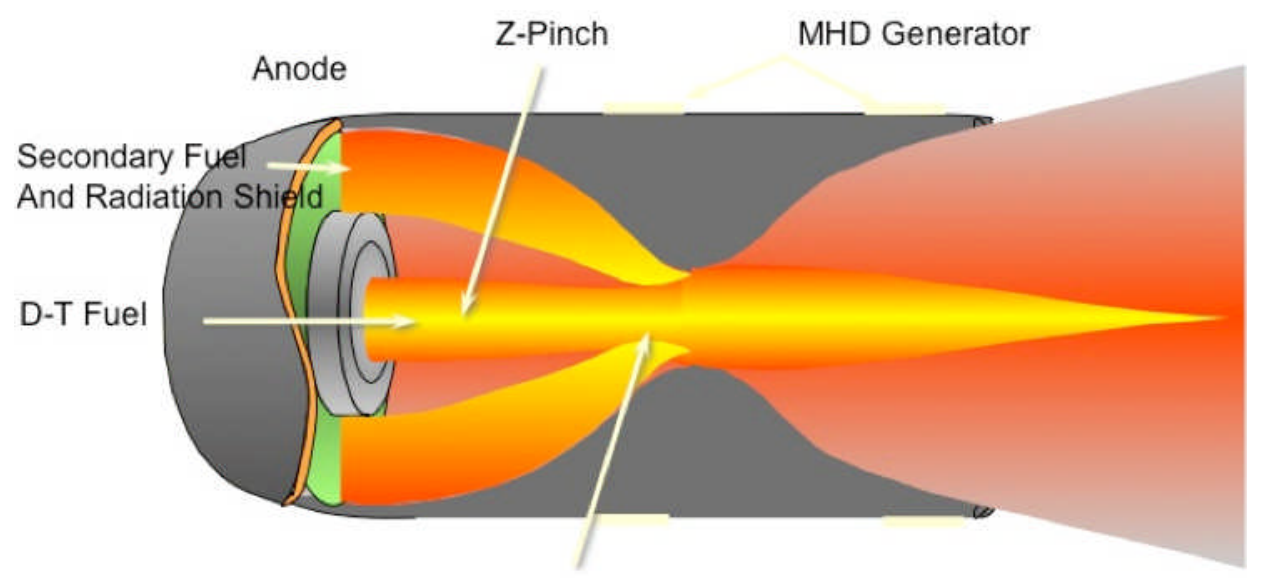

Cathode

Figure 1: Z-Pinch Engine: Physical nozzle shown for illustrative purposes only. 


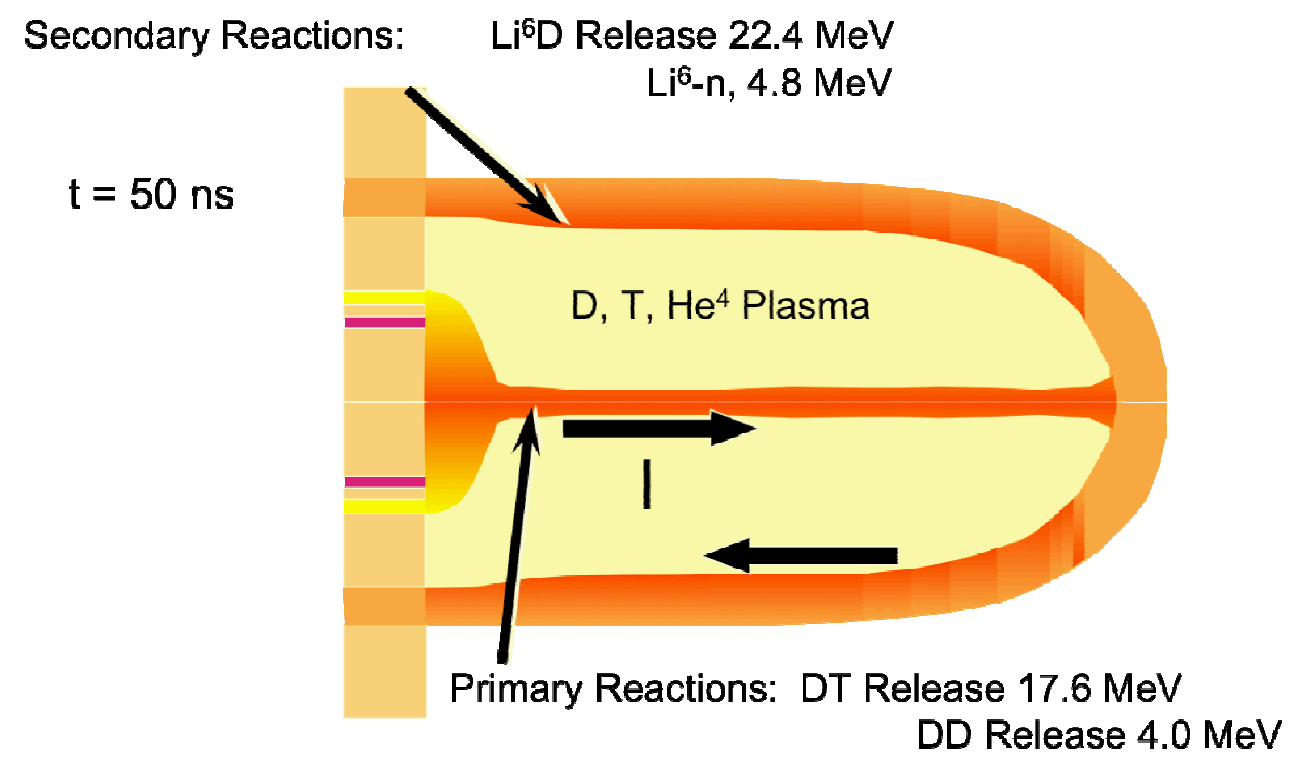

Figure 2: Fusion Ignition Chamber

A Z-Pinch is a radial implosion of a cylindrical or annular plasma under the influence of a strong magnetic field produced by current flowing down the length of the plasma; it usually involves the ionization and subsequent implosion of a gas for time-scales on the order of microseconds. The process can be broken down into a number of steps that occur in the following order (see figure below):

1. Gas injection/pre-ionization

2. Compression/implosion

3. Stagnation/burn

4. Expansion/explosion

Upon close examination, this process can be observed as strikingly similar to the operation of an Otto cycle. For reference, we identify the Otto cycle based on four thermodynamic states numbered 1-4. Process 1-2 is isentropic compression from the cold gas at state 1 to peak compression at state 2. Process 2-3 is constant volume heating. Isentropic expansion follows in process 3-4. Finally, heat transfer returns the system to the original state in process 4-1. Therefore the following correlations can be made:

1) The gas injection/pre-ionization phase can be considered in a similar fashion to the valve intake process of an internal-combustion engine

2) Compression/implosion can be correlated to Process 1-2 of the Otto cycle, making similar assumptions: approximate the plasma as an ideal gas, with constant specific heats and composition.

3) Stagnation/burn is the stage in which the plasma reaches fusion conditions, thus causing fusion reactions to occur. In this case, like the normal combustion process, the fusion reactions can be considered to occur rapidly enough such that the process takes place at constant volume. This stage can therefore be approximated as a constant volume heat addition like Process 2-3 of the Otto cycle.

4) Expansion/explosion, like Process 3-4 of the Otto cycle, can also take place isentropically under the same assumptions. For this particular propulsion application, the process takes place by expanding the plasma out of a magnetic nozzle into a vacuum.

5) Process 4-1 of the Otto cycle can be incorporated into the fusion reaction engine model by assuming constant volume heat rejection from the plasma, thus returning to state 1. Like the Otto cycle, this does not necessarily reflect reality, but is nonetheless useful when considering the general performance of an engine. 
Hypersonic Nozzle

(1) Gas injection \& preionization

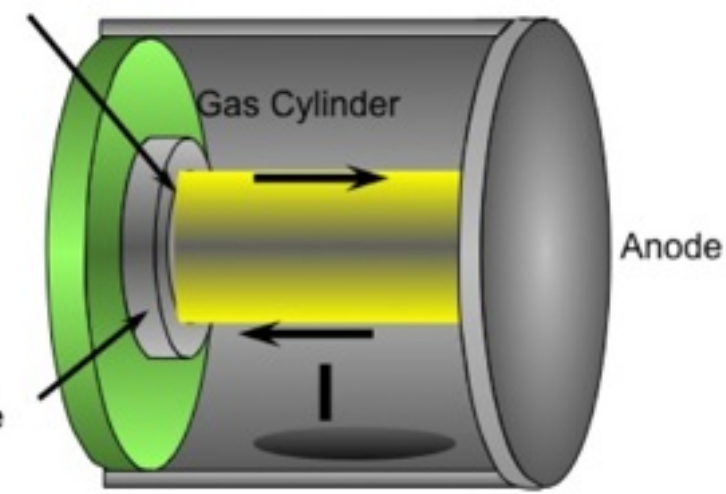

(2) Initial implosion

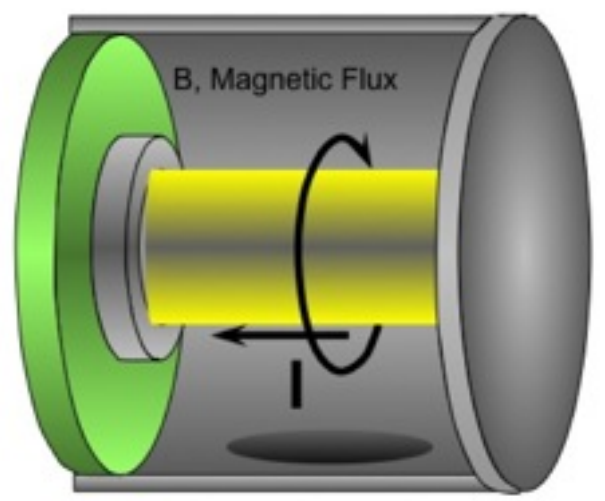

(3) Implosion/stagnation

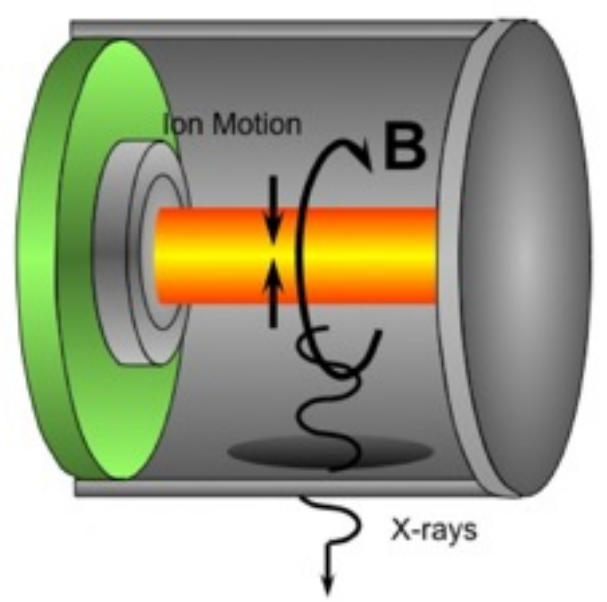

Using the correlations stated above, a useful thermodynamic model incorporating simple plasma physics arguments to describe the performance of a Z-Pinch fusion reaction cycle can be constructed.

\section{Figure 2.4 Stages of Z-Pinch Formation}

\section{Mission Analysis}

For the preliminary trajectory analysis, three missions were assessed. The first was a round trip mission to Mars, where the transfer time between Earth and Mars was set at 90 days. As a sensitivity to this, a 30 day transfer time 
was also assessed. The second mission was a round trip mission to Jupiter. The final mission assessed was a oneway fly-by of a distance $550 \mathrm{AU}$ from the sun. In all cases, the burn-out mass of our vehicle was assumed to be 552 $\mathrm{mT}$, which included payload mass. . The thrust was calculated assuming a $10 \mathrm{~Hz}$ repetition rate and complete thermodynamic equilibration between the lithium liner and fusion fuel. Secondary reactions between lithium and the burning fuel were ignored. Detailed of our fusion model are left in references 3 and 4 . For this study, we selected 38,120 N and a mass flow rate of $0.2 \mathrm{~kg} / \mathrm{sec}$ resulting in 19,400 seconds of specific impulse, consistent with one of the calculated performance points in the model. The results of these missions can be seen in Table 4.1.

Table 4.1 Trajectory Results

\begin{tabular}{|l|c|c|c|c|}
\hline & Mars 90 & Mars 30 & Jupiter & 550 AU \\
\hline Outbound Trip Time (days) & 90.2 & 39.5 & 456.8 & 12936 \\
\hline Return Trip Time (days) & 87.4 & 33.1 & 521.8 & $\mathrm{n} / \mathrm{a}$ \\
\hline Total Burn Time (days) & 5.0 & 20.2 & 6.7 & 11.2 \\
\hline Propellant Burned (mT) & 86.3 & 350.4 & 115.7 & 194.4 \\
\hline Equivalent DV (km/s) & 27.5 & 93.2 & 36.1 & 57.2 \\
\hline
\end{tabular}

The plots in Figures $3-6$ represent the trajectories for each of these cases. Figure 3 shows the outbound and return trajectories for a 90 day trip to Mars. This mission offers a 50\% reduction in the nominal trip time to Mars with a 1.5 day departure burn.
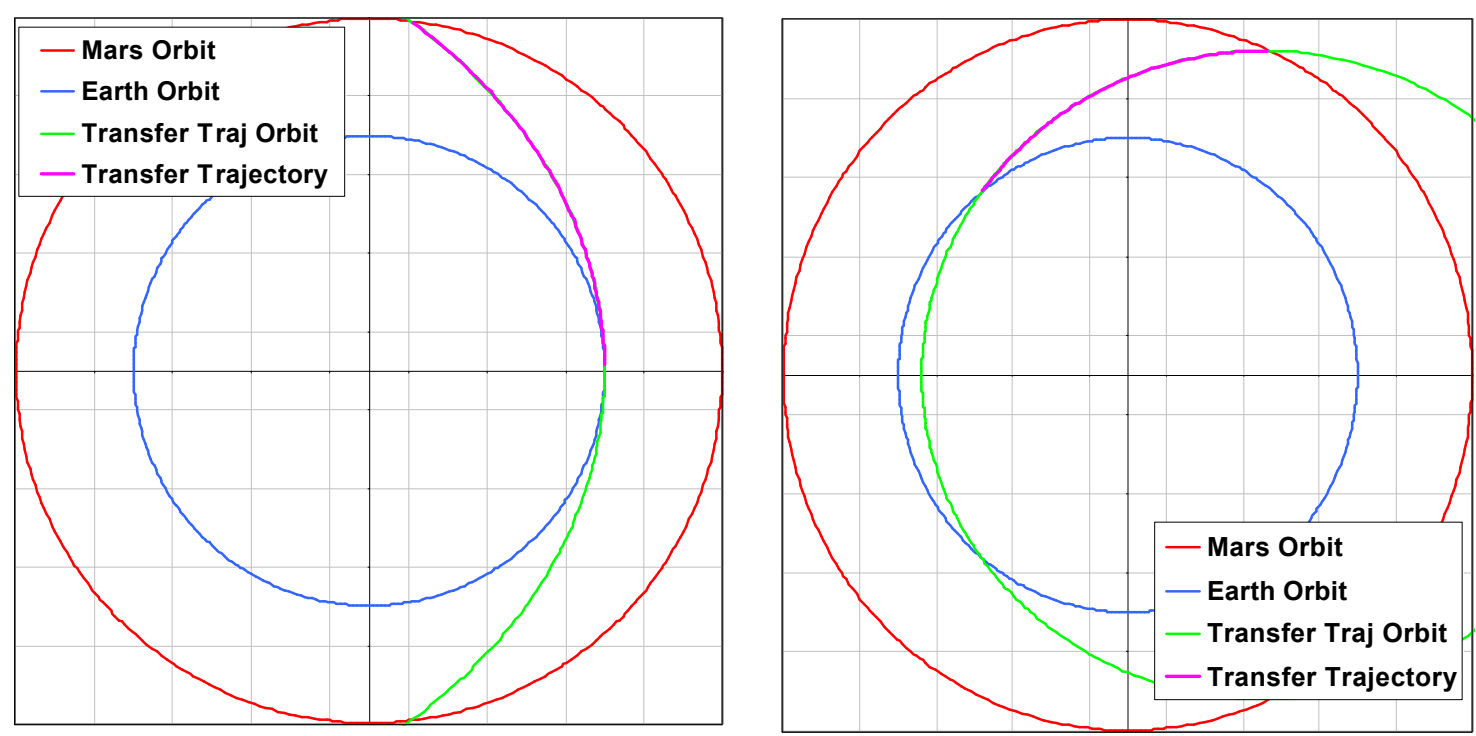

Figure 3 Mars 90 Day Transfer Trajectories

Figure 4 shows one of the integrated Earth departure burns as a representation of all of the integrated burns. In all plots, the burn time is so small compared to the coast time that these burns are not visible on the full trajectory plots.

Figure 5 shows a trajectory from Earth to Jupiter. The goal with this trajectory was to find a closed case with approximately the same propellant load as the Mars vehicle concept. For a propellant load of approximately 116 $\mathrm{mT}$, the outbound trip to Jupiter is 1.25 years and the return trip is 1.42 years.

Figure 6 shows a one way trajectory for a flyby of the region of space $550 \mathrm{AU}$ from the sun. For less than $200 \mathrm{mT}$ of propellant, the spacecraft will fly through the $550 \mathrm{AU}$ mark in 35 years. This gives a speed of roughly $15.7 \mathrm{AU}$ per year, compared to `3.5 Au/year of Voyager 1 and $4.4 \mathrm{AU} /$ year for New Horizions. 


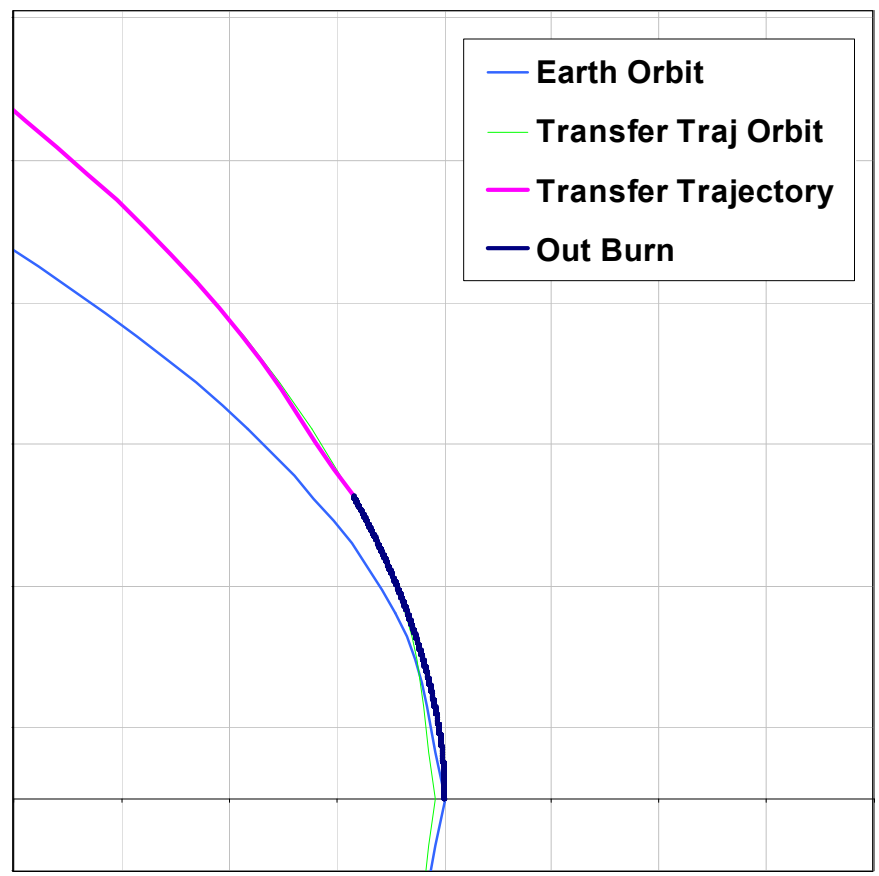

Figure 4 Representative Integrated Earth Orbit Departure Burn
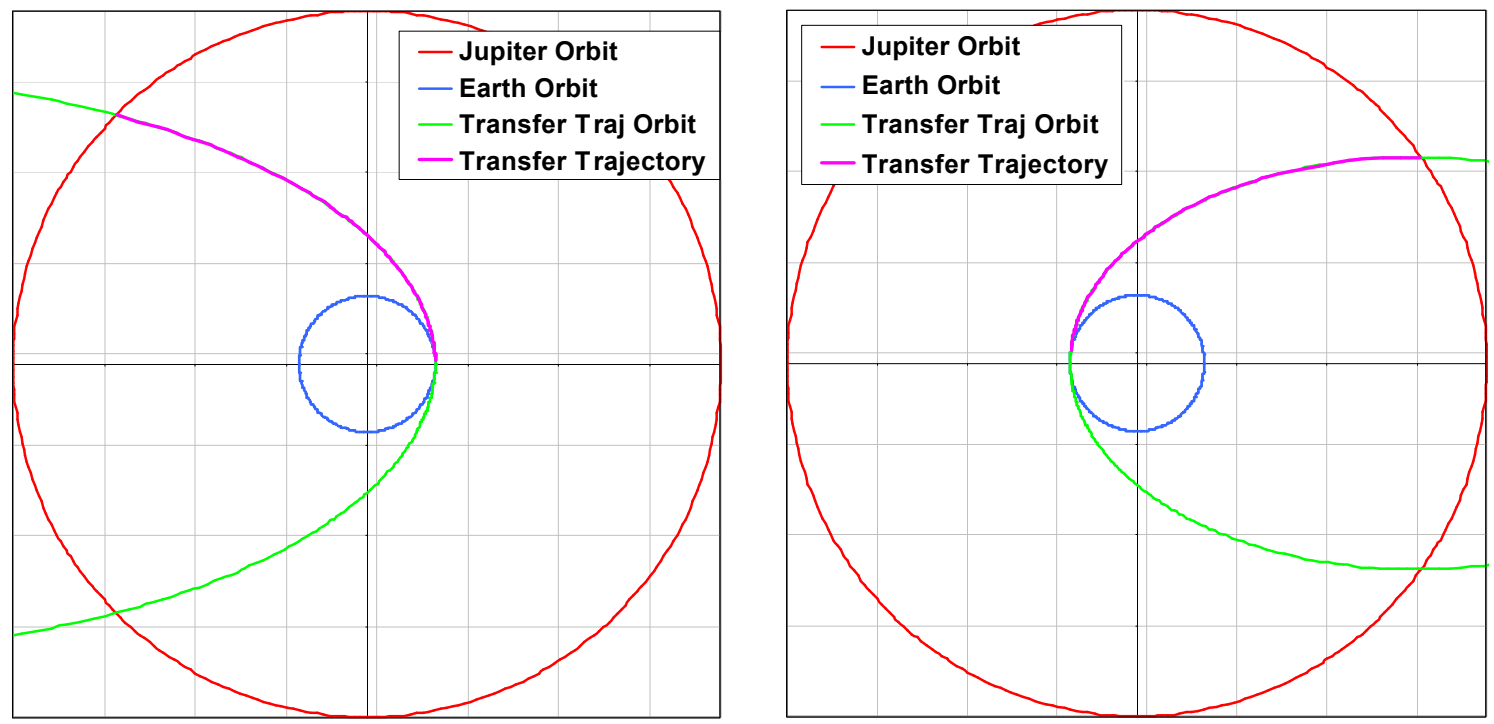

Figure 5 Jupiter Transfer Trajectories

Variations in the design and operational assumptions of the Z-Pinch fusion propulsion system can result in different combinations of thrust and specific impulse. An additional performance point of 55,370 N of thrust and 28,080 seconds of specific impulse was studied. As expected, higher thrust and specific impulse will result in higher payload mass fractions for the same mission. Figure 7 shows the payload mass fractions of the missions studied versus mission Delta-V. The higher data point on each line represents the performance for the higher thrust and specific impulse combination. For perspective, a payload fraction for a traditional system using chemical propulsion and aerobraking at Mars is given. This was calculated from Mars DRA 5 data (Drake, 2009) and corresponds to a 202 day flight time. This figure shows that the Z-Pinch fusion propulsion concept allows for significantly higher delta-v capacity (and shorter trip times) with comparable payload fractions. Figure 8 shows that this propulsion 
system will require significantly lower thrust for faster trips with lower propellant fractions than traditional chemical approaches.

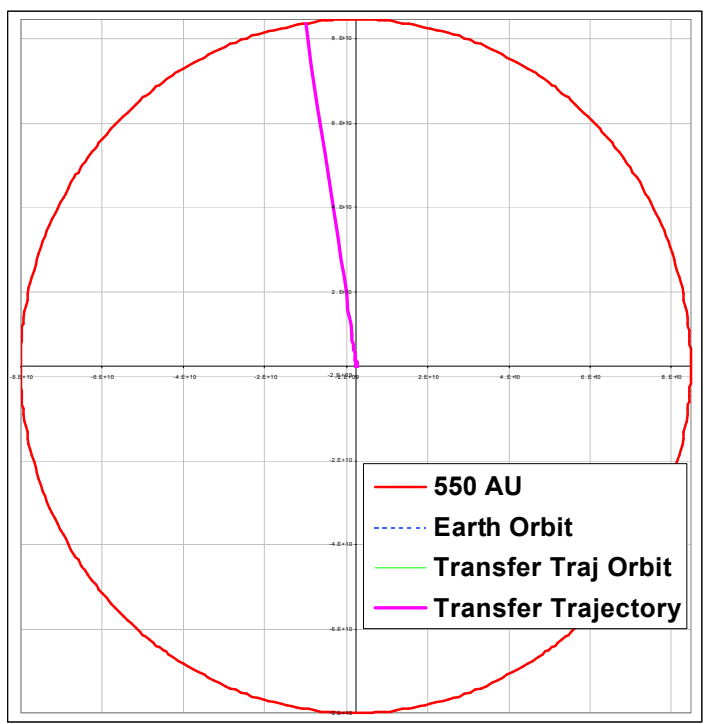

Figure 6 Trajectory to $550 \mathrm{AU}$

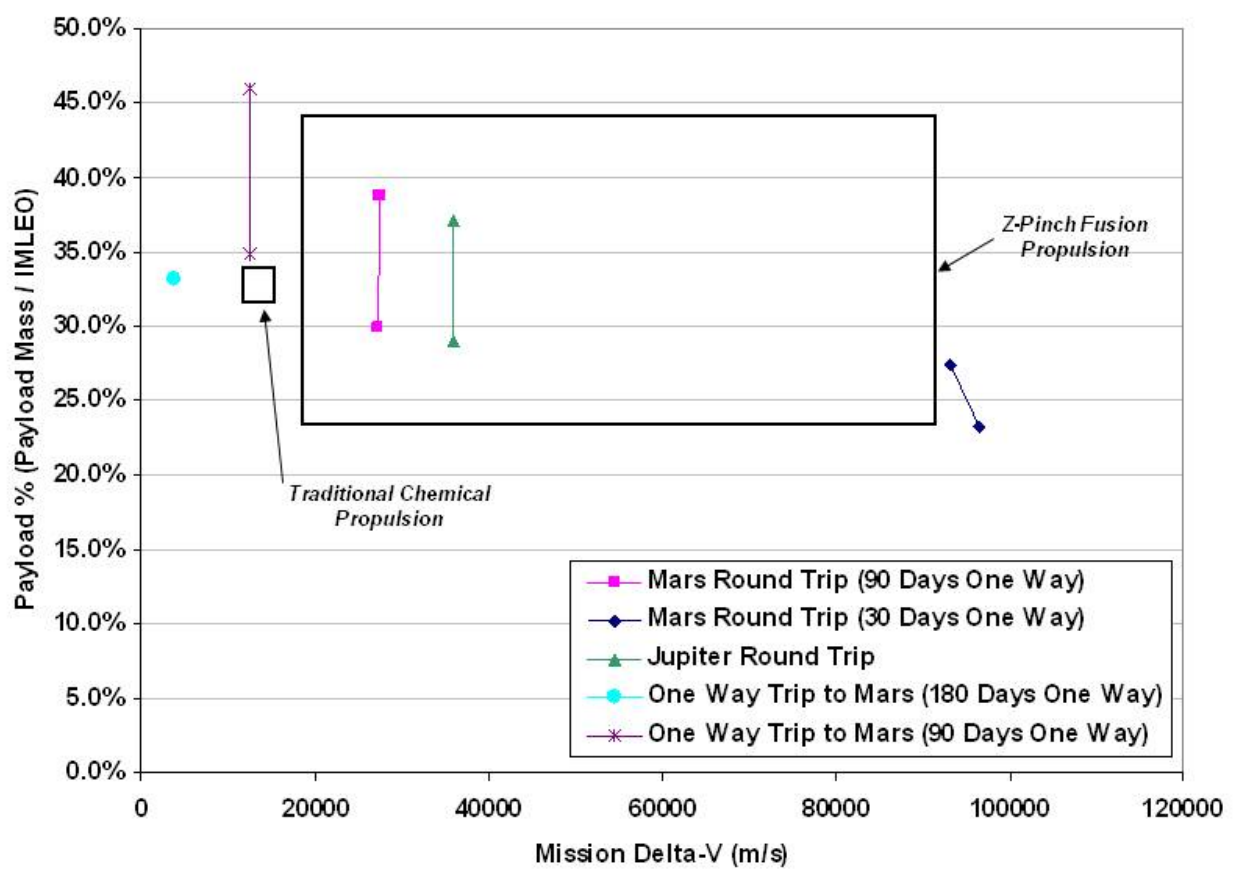

Figure 7 Payload Fractions for Z-pinch Fusion Systems 


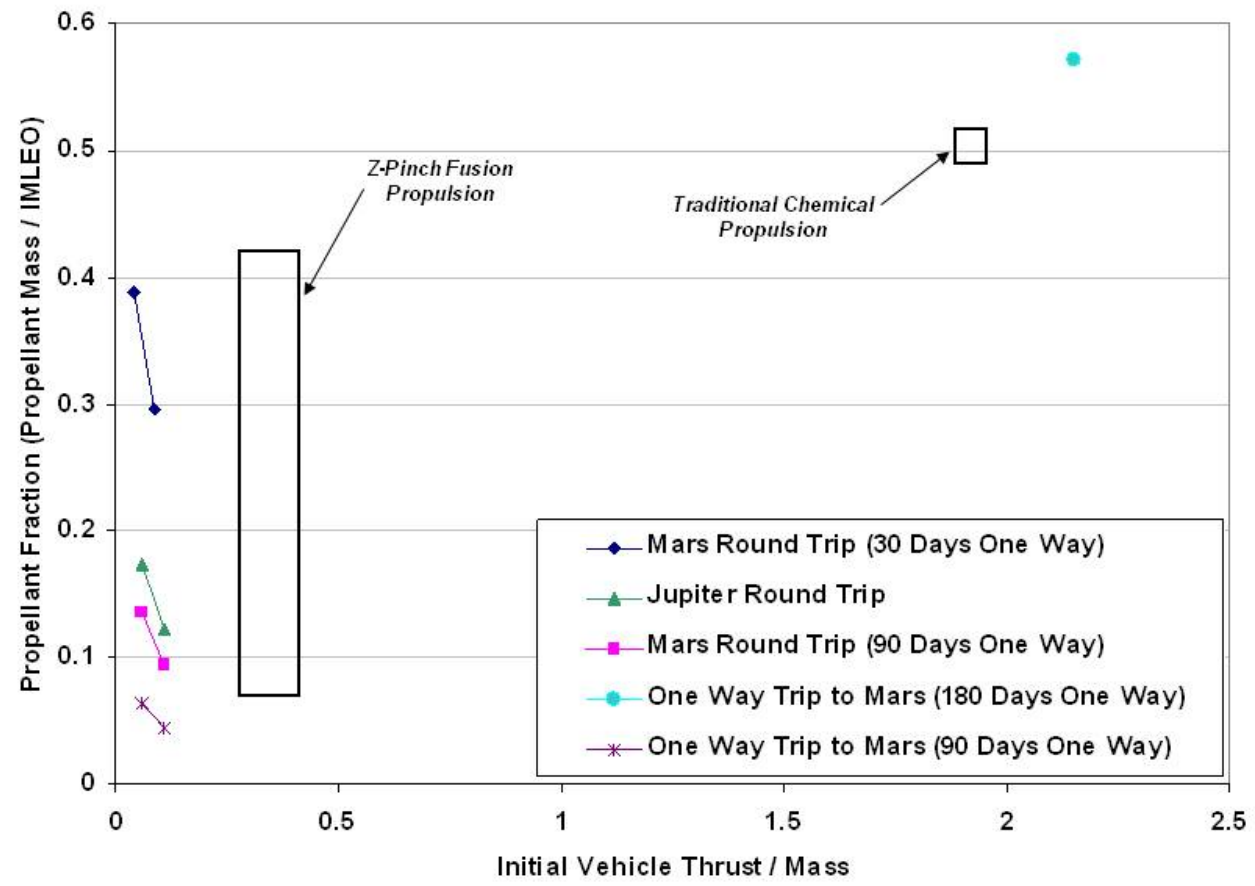

Figure 8 Propellant Mass Fraction vs Thrust/Initial Mass

\section{Vehicle Design}

In order to assess the applicability of Z-Pinch fusion to interplanetary missions, the study team performed a conceptual design of a spacecraft suitable to a number of manned and unmanned missions in the solar system.

The overall design strategy for this vehicle is as follows:

1) The main propulsion system is designed around current Z-Pinch experimental equipment (e.g. the ZMachine's DECADE Marx generator) adapted for flight use along with other components (e.g. magnetic nozzle) developed for other prolusion concepts. The required analytical parameters such as jet power, thrust, specific impulse, thermal output and others are derived from the propulsion models developed in the early phases of the study.

2) The Reaction Control System (RCS) and Thermal Management System are designed with current manned spacecraft technology specifically for this vehicle.

3) The spacecraft power and avionics systems are taken directly from other interplanetary studies so that the vehicle size and performance will be comparable to other interplanetary vehicles developed for similar mission profiles.

4) Spacecraft structures are designed to meet the specific needs of the vehicle and mission.

In comparing this vehicle to other concepts, it should be noted that this is not an optimized design. The time and resource limits for this study required that the designers be conservative in sizing vehicle subsystems, especially when these subsystems required novel approaches and components. Much future work is required to derive flightweight components for the vehicle and as that work is completed, size estimates may be revised to reflect more realistic subsystem masses. Currently, the team's strategy is to be relatively conservative in sizing.

The configuration is shown in Figures 9 and 10. The Vehicle Mass Breakdown is shown in Table 1. 


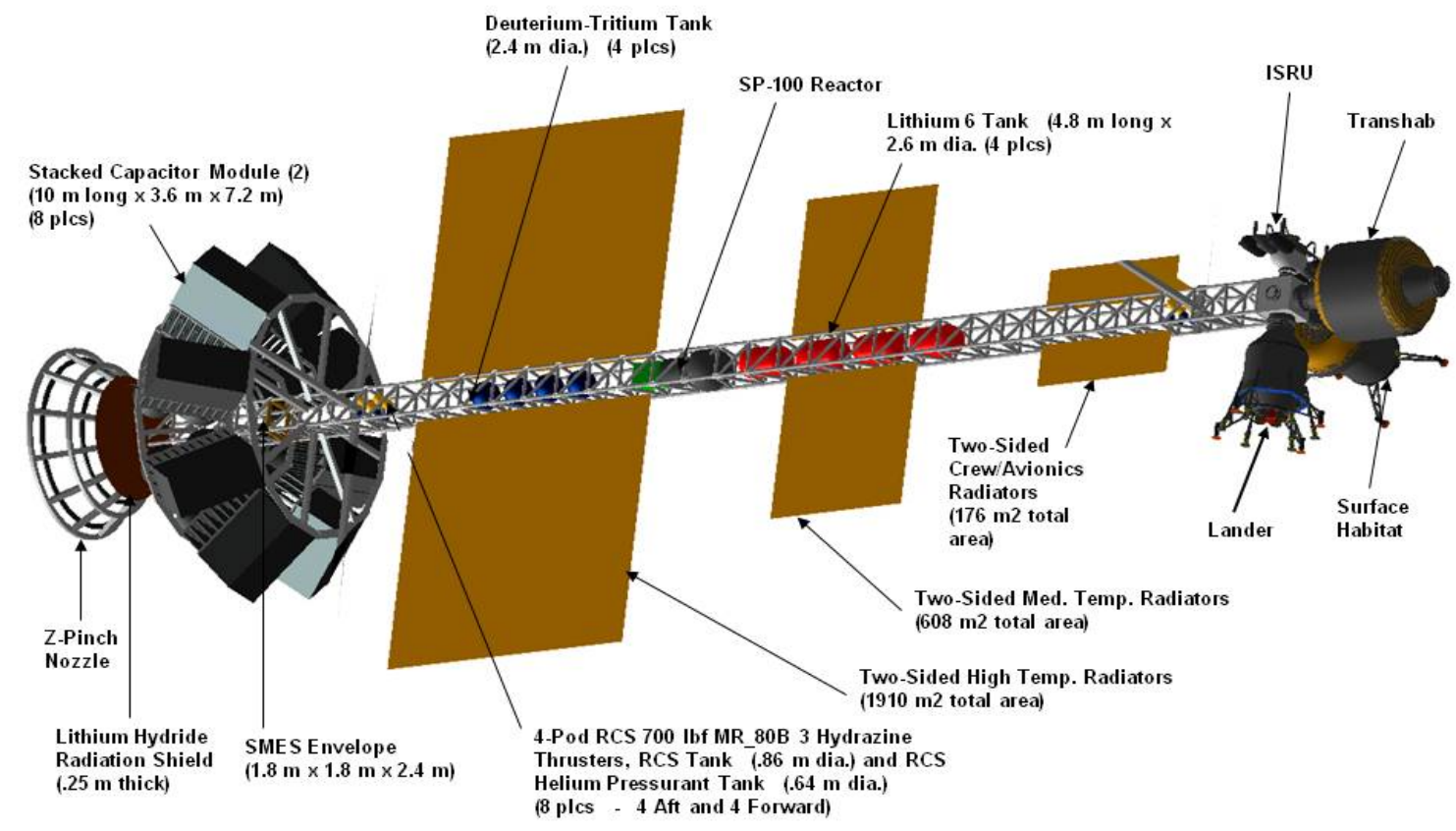

Figure 9 Configuration 1

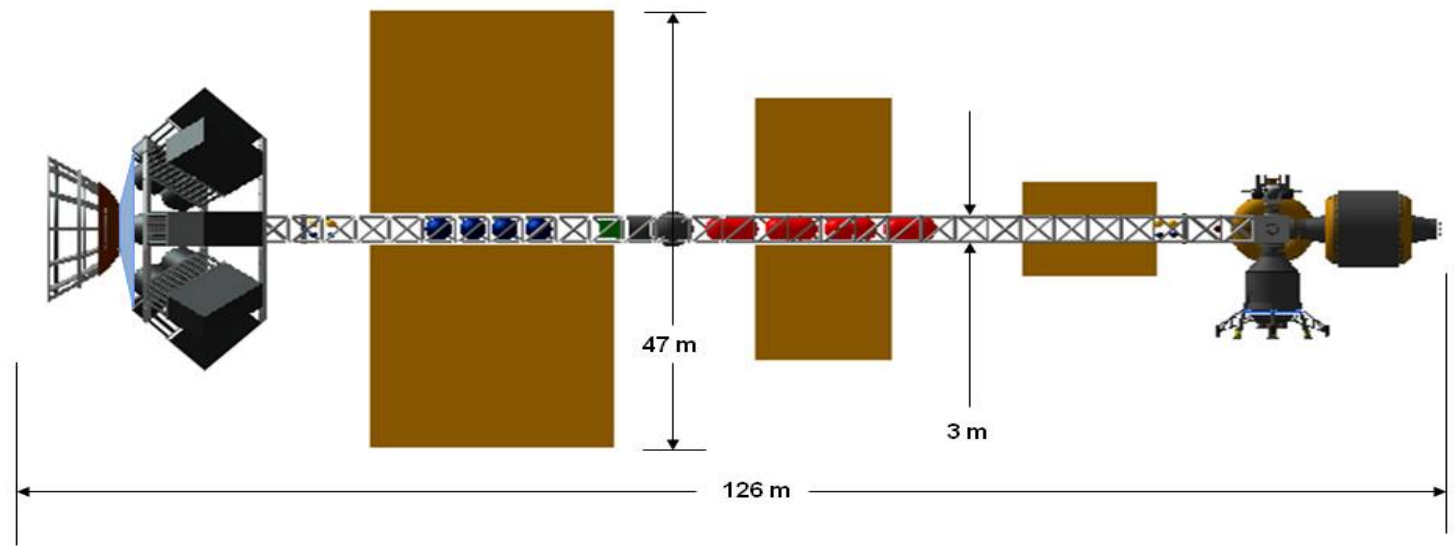

SIDE VIEW

Figure 10 Configuration 2

\section{Conclusions}

A vehicle of this magnitude represents a substantial investment. In order to justify its development, it must be reusable and suitable for a wide variety of missions. It is possible that with some refurbishment between missions, the vehicle could have a very long service life (much like Naval vessels). New (full) propellant tanks could replace the spent ones, and components that incur significant wear and/or damage (Z-Pinch electrical leads, Lithium nozzles, Z-Pinch diodes and switches, SP-100 Reactor, etc) could be replaced while the vehicle is at dock at $\mathrm{L} 1$. 
As the vehicle nears the end of its service life, it could be sent on a very long range one-way mission to an interstellar destination.

Table 1 Vehicle High Level Mass Breakdown

\begin{tabular}{|l|r|}
\hline Subsystem & \multicolumn{1}{|l|}{ Mass $(\mathrm{kg})$} \\
\hline Payload & 150,000 \\
\hline Structures & 54,600 \\
\hline Main Propulsion & 95,138 \\
\hline Reaction Control System & 586 \\
\hline Thermal Management & 77,164 \\
\hline Power & 16,480 \\
\hline Avionics & 389 \\
\hline Total Dry Mass & 394357 \\
\hline 30\% Mass Growth Allowance & 73,307 \\
\hline Total Mass (Best Estimate) & 467,664 \\
\hline
\end{tabular}

\section{Conclusions}

This work was funded in part by the NASA Innovated Partnerships Probram and the Alabama Space Grant Consortium. The authors also thank the NASA Advanced Concepts Office, UAHuntsville and the University of Wisconsin for their cost sharing on this project.

\section{References}

${ }^{1}$ Lindemuth, I., and Siemon, R. (2009). The fundamental parameter space of controlled thermonuclear fusion. American Journal of Physics 77, no. 5: 407-416.

${ }^{2}$ Velikovich, A. L., R. W. Clark, J. Davis, Y. K. Chong, C. Deeney, C. A. Coverdale, C. L. Ruiz, et al. 2007. ZPinch plasma neutron sources. Physics of Plasmas 14, no. 2: 022701. doi:10.1063/1.2435322.

${ }^{3}$ Polsgrove, Tara; Adams, Robert B; Fabisinski, Leo; Fincher, Sharon; Maples, C. Daughne; Miernik, Janie; Percy, Tom; Statham, Geoff; Turner, Matt; Cassibry, Jason; Cortez, Ross; Santarius, John, "Z-pinch pulsed plasma propulsion technology," NASA White Paper, December 2010.

${ }^{4}$ Cortez, R J; Cassibry, J T. "Comparison of Directly and Inductively Coupled Fusion Propulsion Systems". AIAA ASM, Jan. 2011.

${ }^{5}$ Olson, C. e. (2005). Development Path for Z-Pinch IFE. Fusion Science and Technology , 47, 633-640.

${ }^{6}$ Shumlak, U., Lilly, R. C., Adams, C. S., Golingo, R. P., Jackson, S. L., Knecht, S. D., et al. (2006). Advanced Space Propulsion Based on the Flow-Stabilized Z-Pinch Fusion Concept. 42nd AIAA/ASME/SAE/ASEE Joint Propulsion Conference (pp. 1-14). Sacramento, CA: AIAA. 


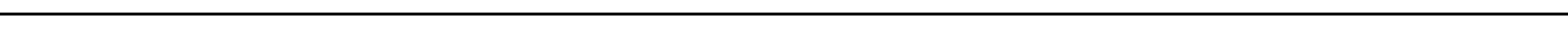

